\title{
ESTUDO DE COMPÓSITOS POLIMÉRICOS DESENVOLVIDOS A PARTIR DE POLIETILENO, TERMOPLÁSTICO DE AMIDO DE MANDIOCA E BIOMASSA FÚNGICA
}

\author{
Clairton Edinei dos Santos $^{1}$ \\ Alex Ketzer ${ }^{2}$ \\ Bruno Bergel ${ }^{3}$ \\ Heliberto José Limberger ${ }^{4}$ \\ Hilton de Souza Rutsatz ${ }^{5}$ \\ Cláudia Mendes Mählmann ${ }^{6}$ \\ Valeriano Antonio Corbellini ${ }^{7}$
}

\section{RESUMO}

Biomassas fúngicas podem ser empregadas para preparação de compósitos poliméricos parcialmente biodegradáveis, sendo uma boa alternativa para diminuição do impacto ambiental gerado pela matriz polimérica. No presente trabalho foram utilizadas fibras de fungos macroscópicos (Picnoporus sp.), coletadas em área de mata nos municípios de Santa Cruz do Sul e Gramado Xavier. Este material foi identificado taxonomicamente e preparado através da limpeza, desidratação em estufa a $50^{\circ} \mathrm{C}$, e trituração. Após foi misturado com polipropileno (PE) e termoplástico à base de amido (TPS). Foram testadas formulações com diferentes proporções de biomassa fúngica $(5,10,15$ e $20 \%)$. As diferentes formulações foram homogeneizadas por extrusão, trituradas e processadas por moldagem por compressão. As propriedades das diferentes amostras foram avaliadas pelos ensaios de tração e flexão. Os resultados obtidos indicaram que a adição de biomassa fúngica acarretou melhora das propriedades mecânicas do material.

Palavras-chave: Compósitos poliméricos. Biomassa fúngica. Fungos macroscópicos.

\section{ABSTRACT}

Fungic biomass can be used to prepare polymeric composites that are partially degradable, this being a good choise to decrease the environmental impact caused by the polymeric matrix. In the current paper were used macroscopic fungi fibers (Picnoporus sp.). They were collected in forests areas of the municipalities of Santa Cruz do Sul and Gramado Xavier. The material was taxonomically identified, processed by washing and dehydration in oven at 50 ${ }^{\circ} \mathrm{C}$ followed by trituration. The material was then mixed with polypropylene and thermoplastic starch. Four different formulations were tested, containing 5, 10, 15 and $20 \%$ of fungic biomass. The formulations were homogeneized by extrusion followed by trituration and processed by hot plate molding. The sample properties were evaluated by tensile and

\footnotetext{
${ }^{1}$ Aluno de Ciências Biológicas da Universidade de Santa Cruz do Sul - UNISC. <clairton03@ hotmail.com>

${ }^{2}$ Aluno de Química Industrial da Universidade de Santa Cruz do Sul - UNISC. $\leq$ alexreztek@ hotmail.com>

${ }^{3}$ Aluno de Química Industrial da Universidade de Santa Cruz do Sul - UNISC. 〈bruno-bergel@ hotmail.com>

${ }^{4}$ Aluno de Química Industrial da Universidade de Santa Cruz do Sul - UNISC. $<$ heliberto.limberger@hotmail.com>

${ }^{5}$ Aluno de Engenharia Mecânica da Universidade de Santa Cruz do Sul - UNISC. <hiltonrutsatz@gmail.com>

${ }^{6}$ Docente do Departamento de Química e Física da Universidade de Santa Cruz do Sul - UNISC. <mclaudia@unisc.br>

${ }^{7}$ Docente do Departamento de Química e Física da Universidade de Santa Cruz do Sul - UNISC. <valer@unisc.br>
} 
flexural tests. The results pointed that there is an increase in mechanic properties by adding biomass.

Keywords: Fungic biomass. Polymeric composites. Macroscopic fungi.

\section{INTRODUÇÃO}

A busca crescente por produtos e materiais diferenciados e ambientalmente corretos torna imprescindível a realização de estudos para o desenvolvimento de materiais parcial ou totalmente degradáveis, visando a diferentes aplicações. Uma alternativa de material parcialmente degradável é a blendagem de uma poliolefina com algum polímero natural, aumentando, dessa forma, a biodegradabilidade do produto obtido. Um dos polímeros biodegradáveis muito estudado atualmente é o obtido a partir de amido, que apresenta baixo custo e fácil obtenção.

As fibras naturais também podem ser incorporadas à massa polimérica, obtendo-se compósitos e possibilitando ganho de suas propriedades. As fibras naturais podem ser obtidas das mais diversas maneiras. No presente trabalho foram utilizadas fibras oriundas de basidiocarpos de fungos macroscópicos coletados em área de mata nos municípios de Santa Cruz do Sul e Gramado Xavier. Neste estudo estão apresentados os resultados alcançados nos testes utilizando polietileno e termoplástico à base de amido de mandioca como matriz polimérica parcialmente degradável, com biomassa fúngica de Picnoporus sp. como fase dispersa, almejando aprimorar as características do compósito.

\section{FUNDAMENTAÇÃO TEÓRICA}

Esta seção apresenta os conceitos relacionados às fibras naturais e termoplástico de amido.

\subsection{Fibras naturais}

As fibras naturais estão sendo apontadas como soluções para a produção de compósitos poliméricos e a sua utilização gera ganhos econômicos e ambientais, uma vez que 
oferece mercado para resíduos fibrosos e propicia a utilização de material biodegradável para confecção de compósitos (ISHISAKY et al., 2006).

Estudos indicam que as fibras naturais muitas vezes apresentam propriedades mecânicas próximas às fibras convencionais, considerando que elas possuem boas propriedades de isolamento térmico, elétrico e acústico. Além disso, são facilmente obtidas e a um custo relativamente baixo. (DUC et al., 2011)

A utilização de biomassa fúngica obtida através de uma fonte natural, que no presente trabalho é a biodiversidade local, para preparação de compósitos é uma boa alternativa para a redução de impacto ambiental gerado pela matriz polimérica.

\subsection{Termoplástico de amido}

O termoplástico de amido (TPS) é um polímero relativamente novo, apresentando propriedades interessantes que justificam o grande interesse de pesquisas relacionadas à sua utilização. Para a produção de TPS faz-se necessário o uso de plastificantes, uma vez que, depois da gelatinização, o amido não pode voltar a formar ligações de hidrogênio (TEIXEIRA et al., 2012).

Estudos têm demonstrado que a quantidade de plastificante utilizado na gelatinização afeta diretamente a viscosidade do termoplástico fundido. Deste modo, modificando a concentração do plastificante podem-se utilizar diferentes técnicas de processamento, tais como injeção, extrusão e moldagem por compressão (RODRIGUEZ-GONZALEZ et al., 2004).

Como o TPS possui uma grande solubilidade em água, somando-se às propriedades mecânicas pobres deste, faz-se necessário o emprego de técnicas que visam a minimizar estes efeitos; uma delas é a blendagem (SCHLEMMER, 2007).

O polietileno é amplamente utilizado pela sociedade, gerando consequentemente uma grande quantidade de resíduos e, mesmo sendo possível a sua reciclagem, muitas vezes a aplicação deste em certos produtos só é possível quando se encontra no estado virgem.

A blendagem é um processo comumente utilizado para a obtenção de misturas que apresentem características intermediárias entre os polímeros base. A miscibilidade entre os polímeros nem sempre é alcançada, necessitando a utilização de diferentes técnicas de blendagem, como a por solvent casting. 
Blendas formadas a partir de poliolefinas e polímeros biodegradáveis apresentam biodegradabilidade elevada devido principalmente ao contato de vários grupos funcionais hidroxila (RAMIS et al., 2004).

A produção de compósitos com fibras naturais estão cada vez conquistando mais espaço, uma vez que permitem um aumento na resistência do produto final e, como as fibras naturais são de baixa densidade, proporcionam também uma diminuição do peso final do compósito. Alguns estudos têm demonstrado que a adição de fibras naturais para produção de compósitos utilizando TPS como matriz incrementam as propriedades mecânicas, aumentam a resistência à absorção de água devido à boa interação das fibras com o TPS. Logo, a associação de matriz com TPS e as fases dispersas de fibras naturais despertam interesse devido aos fatores: baixo custo, biodegradabilidade, aparência visual, entre outros (YU et al., 2006).

\section{METODOLOGIA}

\subsection{Reagentes e equipamentos}

Para o desenvolvimento deste trabalho foram utilizados polietileno (PE), amido de mandioca, glicerol Synth 99,5\% de pureza.

Os equipamentos utilizados na realização deste trabalho foram: forno de micro-ondas convencional (Panasonic, potência de $2450 \mathrm{MHz}, 1100 \mathrm{~W} / 1600 \mathrm{~W}$ ); moinho de facas Mecanofar - MF300; extrusora monorrosca SEIBT; homogeneizador de Laboratório MH-100 (MH Equipamentos); prensa hidráulica (MH Equipamentos); balança analítica (Quimis); estufa De Leo; microrretífica (DREMEL); máquina Universal de Ensaios Mecânicos (EMIC DL 10.000) e acessórios.

\subsection{Produção de termoplástico de amido}

O termoplástico de amido (TPS) foi produzido através da gelatinização de uma mistura de 16:4:80 (m/m/m) de amido de mandioca, glicerol e água. A gelatinização se deu em micro-ondas convencional em potência média, com intervalos de 1 minuto para homogeneização. $\mathrm{O}$ gelatinizado foi seco em estufa a uma temperatura de $75-80^{\circ} \mathrm{C}$. $\mathrm{O}$ produto seco foi triturado e armazenado. 


\subsection{Produção da matriz (blendas)}

Foram elaboradas blendas de polietileno e TPS em diferentes proporções para que fosse permitido observar a influência do TPS em cada blenda. Os materiais foram misturados em homogeneizador, triturados em moinho de facas e, com os flakes obtidos, foram produzidas placas por moldagem por compressão a quente. Das placas foram obtidos os corpos de prova para os ensaios de tração e flexão.

\subsection{Ensaios de caracterização das propriedades}

Para analisar as propriedades das amostras processadas foram realizados ensaios descritos como segue:

- Tração: Foi realizado seguindo procedimento descrito na norma ASTM D638. Os ensaios foram realizados na máquina universal de ensaio EMIC DL 10.000, com velocidade do ensaio de $5 \mathrm{~mm} \mathrm{~min}^{-1}$.

- $\quad$ Flexão: Foi realizado seguindo procedimento descrito na norma ASTM D790, na máquina universal de ensaio EMIC DL 10.000, com velocidade do ensaio de $5 \mathrm{~mm} \mathrm{~min}^{-1}$.

\subsection{Obtenção da fibra fúngica}

Basidiocarpos de Picnoporus sanguimeus foram coletados e, depois de encaminhados para o laboratório e lavados, foram desidratados (mínimo de $48 \mathrm{~h}$ em temperatura ambiente), triturados e peneirados com malha de $4 \mathrm{~mm}$ para obter um padrão no tamanho das fibras.

\subsection{Produção dos compósitos}

Utilizamos os teores de 5, 10, 15 e $20 \%$ de biomassa fúngica para $70 \%$ de polietileno e 30\% de TPS. Após, o material extrusado foi moído, em moinho de facas, e processados na prensa para obtenção dos corpos de prova para a realização dos ensaios de caracterização de propriedades físico-mecânicas. 


\section{RESULTADOS E DISCUSSÕES}

\subsection{Produção da matriz (blendas)}

A biomassa fúngica moída foi misturada com PE+TPS e logo após extrusada visando à homogeneização do compósito. Durante a extrusão, o fungo utilizado alterou sua cor característica, inicialmente vermelha, ficando com aspecto mais escuro, possivelmente devido à temperatura empregada durante o processamento. A Figura 1 mostra a cor característica do fungo antes da extrusão e o aspecto obtido após mistura e processamento com os termoplásticos na extrusora.

Figura 1 - Aspecto visual da biomassa fúngica moída na coloração vermelha antes da extrusão (A) e aspecto após extrusão e homogeneização com os termoplásticos (B)

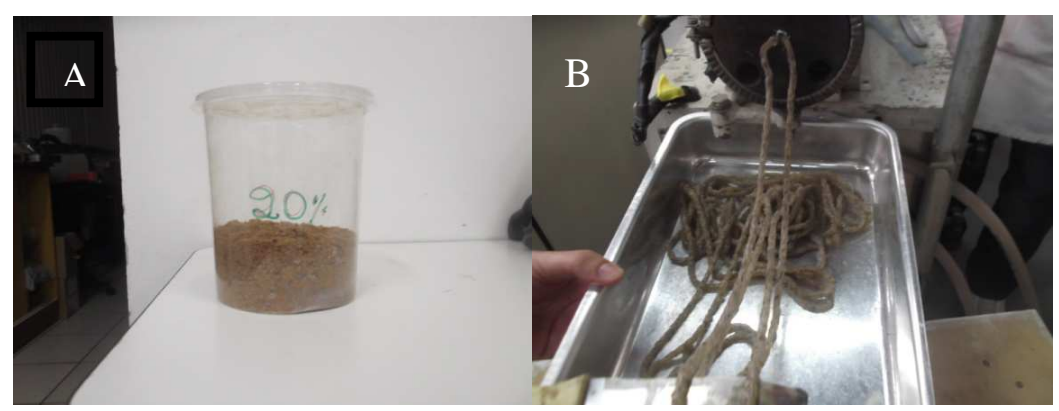

O material, após ser homogeneizado/extrusado, foi triturado em moinho de facas, processado através de moldagem por compressão a quente, obtendo-se placas de espessura 4 $\mathrm{mm}$. Após o resfriamento do material à temperatura ambiente, realizou-se o desenho e corte dos corpos de prova, como apresentado na Figura 2.

Figura 2 - Preparação do material moído no molde para processamento através de moldagem por compressão (A). Placa de compósito obtido, com o desenho dos corpos de prova (B).

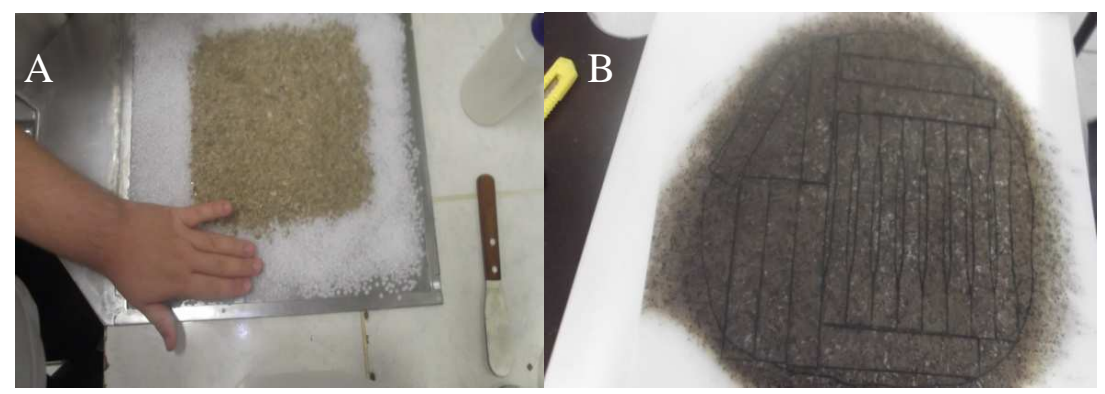




\subsection{Ensaios de caracterização das propriedades}

Após realização dos ensaios de caracterização das amostras, utilizando a máquina universal de ensaio EMIC DL 10.000, os resultados obtidos foram organizados por ensaio e parâmetro avaliado. Os resultados obtidos através do ensaio de flexão são apresentados para o módulo de elasticidade na Figura 3 e para tensão na força máxima na Figura 4.

Figura 3 - Resultados do ensaio de flexão para o parâmetro módulo elasticidade de compósito com diferentes proporções de biomassa de Picnoporus sanguineus (picno)

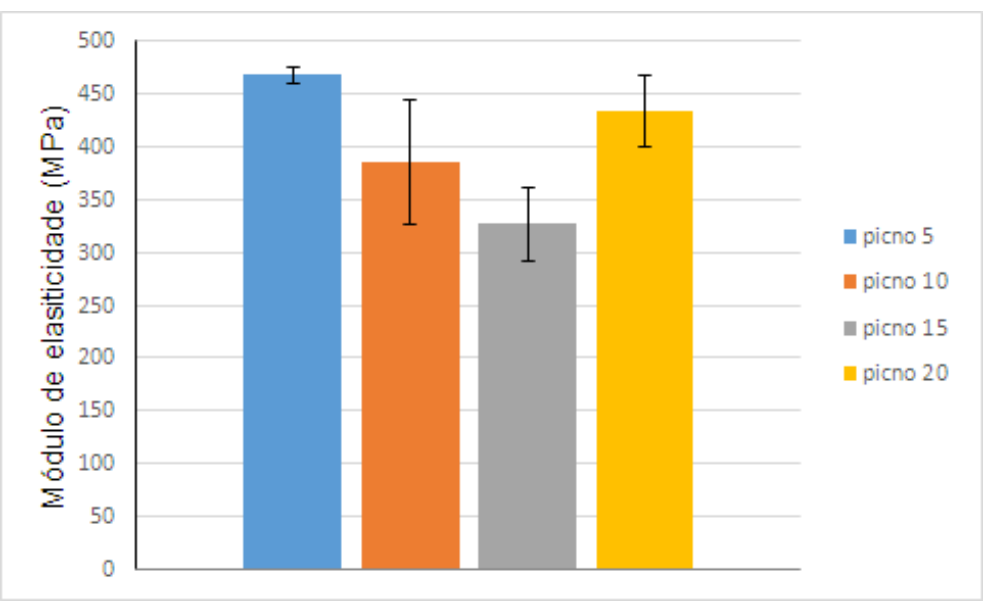

Como o módulo de elasticidade vincula-se à rigidez do material, observou-se que a rigidez dos compósitos em flexão diminuiu com o aumento da concentração de biomassa fúngica na composição para até $15 \%$ de biomassa. Para o compósito com $20 \%$ de biomassa fúngica, observou-se aumento da rigidez.

Figura 4 - Resultados do ensaio de flexão para o parâmetro tensão na força máxima

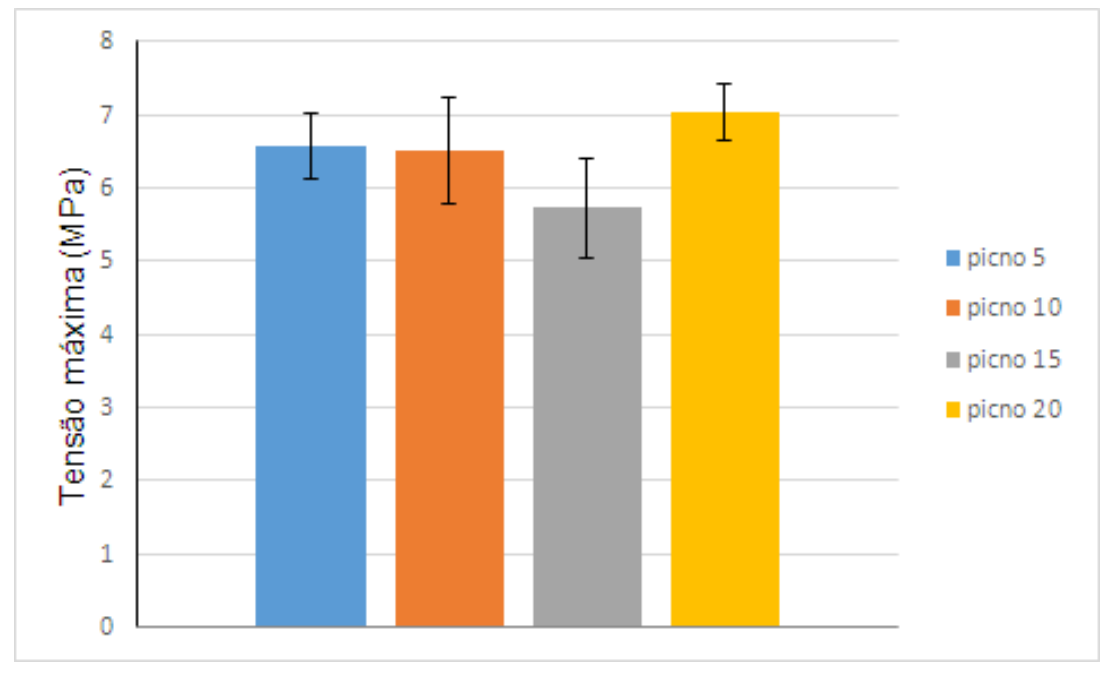


Para a tensão na força máxima verificou-se o comportamento análogo ao do módulo de elasticidade. Inicialmente, houve uma perda de resistência mecânica com aumento de teor de fase dispersa (biomassa fúngica), até $15 \%$. E para a amostra de maior quantidade de biomassa (20\%) houve um ganho de resistência.

\subsection{Tração}

Os resultados obtidos de módulo de elasticidade do ensaio de tração dos compósitos de PE/TPS/fibras fúngicas são mostrados no gráfico da Figura 5. Observou-se que a amostra com $5 \%$ de biomassa fúngica apresentou maior rigidez, sendo que as demais amostras apresentaram uma queda brusca no módulo de elasticidade.

\section{Figura 5 - Resultados do módulo de elasticidade do ensaio de tração}

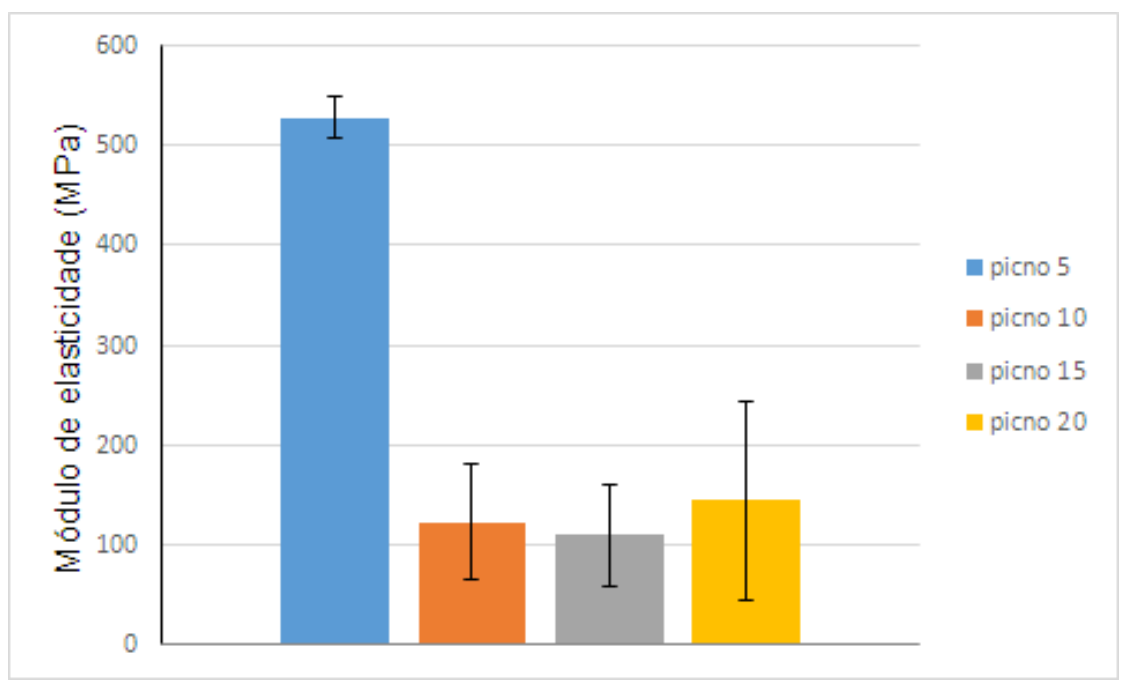

Verificou-se a diminuição da resistência mecânica com o aumento de concentração de biomassa fúngica nas composições (Figura 6).

Carr et al (2006) investigaram quantidade e forma (pó ou fibra) da fase dispersa de origem vegetal utilizadas na formulação de compósitos baseados em amido de mandioca.e encontraram que a forma não indicou melhora nas propriedades. As amostras avaliadas com menor teor de fase dispersa apresentaram melhores resultados reforçando efetivamente os compósitos estudados.

Os compósitos em geral apresentaram boas propriedades para a formulação com $5 \%$ de fase dispersa (biomassa fúngica). Ainda, a menor quantidade deste componente diminui a 
probabilidade de absorção de umidade, bem como garante boa homogeneização dos componentes durante o processamento.

\section{Figura 6 - Resultados de tensão na força máxima do ensaio de tração}

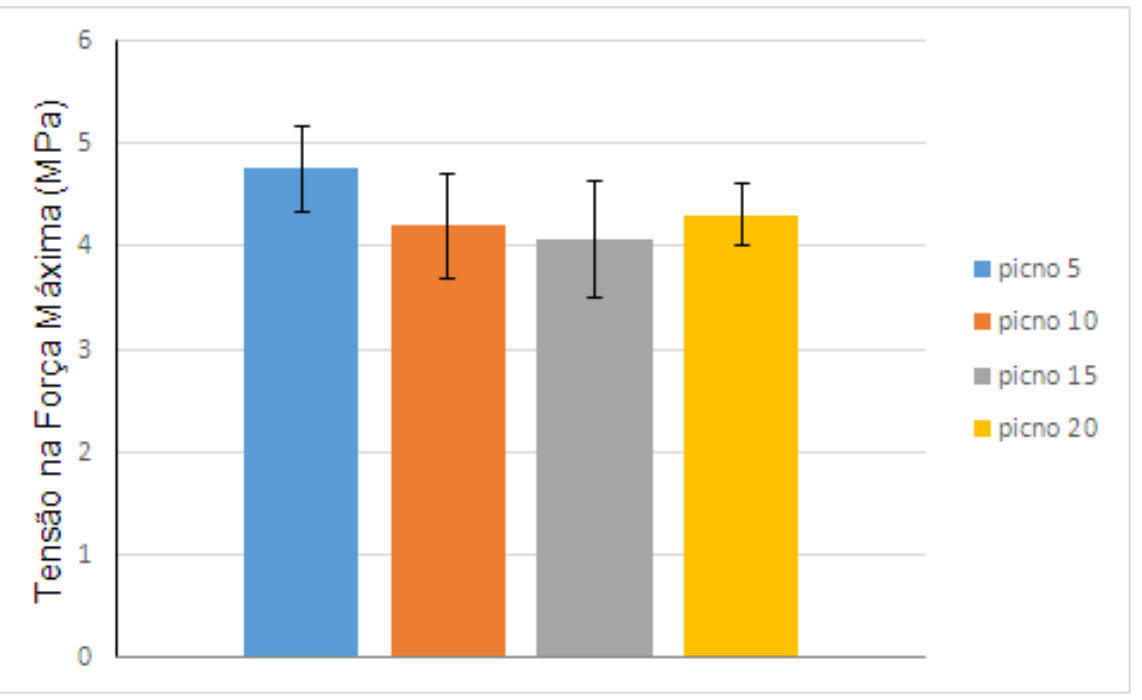

Alguns artigos, inclusive de revisão (AVÉROUS et al., 2009), citam compósitos envolvendo termoplástico baseados em amido reforçados com fibras naturais considerando-se macro-biocompósitos ou nano-biocompósitos, mas não foi encontrado qualquer trabalho publicado que tenha envolvido a utilização de fungos como fase dispersa.

\section{CONCLUSÃO}

Através dos resultados obtidos é possível concluir que a utilização da mistura PE + TPS acrescida de 5\% de biomassa fúngica é a mais recomendada para a preparação do compósito, uma vez que possui uma quantidade razoável de polímero biodegradável e conserva grande parte das propriedades mecânicas.

Os compósitos produzidos a partir da adição de biomassa fúngica apresentaram melhoras nas propriedades do material. Observou-se, também, que os compósitos contendo 10, 15 e $20 \%$ de biomassa fúngica apresentaram resultados ligeiramente menores do que o compósito com 5\%, indicando a perda de propriedades mecânicas associada à quantidade de fase dispersa utilizada. Este tipo de compósito é considerado parcialmente miscível, o que indica a possibilidade de melhora dos resultados através do uso de algum agente compatibilizante. 


\section{REFERÊNCIAS}

AVÉROUS, L.; HALLEY, P. J. Biocomposites based on plasticized starch. Biofuels, Bioproducts and Biorefining, v. 3, n. 3, p. 329-343, 2009.

CARR, L. G.; PARRA, D. F.; PONCE, P.; LUGÃO, A. B.; BUCHLER, P. M. Influence of Fibers on the Mechanical Properties of Cassava Starch Foams. Journal of Polymers and the Environment, v. 14, n. 2, p. 179-183, 2006.

DUC, A. L.; VERGNES, B.; BUDTOVA, T. Polypropylene/nature fibres composites: Analysis of fibre dimensions after compounding and observations of fibre rupture by rheooptics. Composites: Part A, n. 42, p. 1727-1737, 2011.

ISHIZAKI, M. H.; FURTADO, C. R. G.; LEBLANC, J. L. Caracterização Mecânica e Morfológica de Compósitos de Polipropileno e Fibras de Coco Verde: Influenciado Teor de Fibra e das Condições de Mistura. Polímeros: Ciência e Tecnologia, v. 16, n. 3, p. 182-186, 2006.

MA, X.; YU, J.; KENNEDY, J. F. Studies on the properties of natural fibers-reinforced thermoplastic starch composites. Carbohydrates polymers, n. 62, p. 19-24, 2005.

RAMIS, X.; CADENATO, A.; SALLA, J. M.; MORANCHO, J. M.; VALLE'S, A.; CONTAT L., RIBES, A. thermal degradation of polypropylene/stach-basesd materials with enhanced biodegradability. Polymer Degradation and Stability, n. 86, p. 483-491, 2004.

RODRIGUEZ-GONZALEZ, F. J.; RAMSAY, B. A.; FAVIS, B. D. Rheological and thermal properties of thermoplastic starch with high glycerol content. Carbohydrate Polymers, n. 58, p. 139-147, 2004.

SCHLEMMER, D. Preparação, caracterização e degradação de blendas de poliestireno e amido termoplástico usando glicerol e óleo de buriti (Mauritia flexuosa) como plastificantes. Dissertação (Programa de Mestrado em Química) Instituto de Química - Unb Brasília, 2007.

SOBCZAK, L. L. R. W.; HAIDER, A. A. Polypropylene composites with natural fibers and wood - general mechanical property profiles. Composites Science and Technology, v. 72, n. 5, p. 550-557, 2012.

SPINACÉ, M. A. S.; DE PAOLI, M. A. A. tecnologia da reciclagem de polímeros. Química Nova, v. 28, n. 1, p. 65-72, 2005.

TEIXAIRA, E. D. M.; CURVElO, A. A. S.; CORRÊA, A. C.; MARCONCINI, J. M.; GLENN, G. M.; MATTOSO, L. H. C. Properties of thermoplastic starch from cassava bagasse and cassava starch and their blends with poly (latic acid). Industrial Crops and Products, v. 37, n. 1, p. 61-68, 2012.

YU, L.; DEAN, K.; LI, L. Polymer blends and composites from renewable resources. Process in Polymer Science, n. 31, n. 6, p. 576-602, 2006. 